Pak. j. sci. ind. res. Ser. B: biol. sci. 2016 59(2) 75-79

\title{
Comparative Efficacy of Some Quinolones and Doxycycline Against Chronic Infection of Brucella melitensis 16M in BALB/c Mice
}

\author{
Mazen Safi*, Bassam Albalaa, Nermen Haj Mahmoud and Ayman Al-Mariri \\ Department of Molecular Biology and Biotechnology, Atomic Energy Commission, \\ P.O. Box 6091, Damascus, Syria
}

(received April 8, 2015; revised December 3, 2015; accepted December 22, 2015)

\begin{abstract}
This study was under taken to observe various treatment methods for brucellosis caused by Brucella melitensis. The effect of therapeutic regimens with ciprofloxacin, ofloxacin and levofloxacin alone or in combination with doxycycline was assessed against $B$. melitensis chronic infection using 200 mice. Doxycycline alone or in combination with ciprofloxacin was significantly found to reduce the infection till 135 days post-infection $(\mathrm{p}<0.0001)$. Moreover, doxycycline was more effective than ciprofloxacin and ofloxacin 135 days post-infection $(\mathrm{p}=0.04$ and $\mathrm{p}=0.02$, respectively). However, treatment with quinolone-doxycycline combinations revealed synergistic effects as they were able to reduce the splenic cell forming unit (CFU) from day 45 post-infection. Similarly, doxycycline treatment reduced the splenic colony forming unit (CFU) from day 90 post-infection. In conclusion, doxycycline seems to be the most effective agent against Brucella chronic infection.
\end{abstract}

Keywords: doxycycline, quinolones, Brucella melitensis, BALB/c mice

\section{Introduction}

Brucellosis remains the most common and serious problem in some parts of the world (Pappas et al., 2006). Ingestion of unpasteurized dairy products, as well as occupational exposure to infected animals are the major causes of brucellosis. Many Brucella species infect animals through direct contact. However, human could be infected when exposed to B. abortus, B. melitensis, or $B$. suis and the exhausting disease could become, over time, a chronic disease that affects several organs. In addition, some species could be used in a bioterrorist attack (Ariza et al., 2001).

In ruminants, no symptoms were seen after the first abortion; but these infected animals might develop a chronic disease, become chronic carrier, and Brucella could be found in milk and uterine discharges of these carriers during subsequent pregnancies (Díaz et al., 2006).

In humans, undulant fever recovers completely within three to twelve months with only few patients suffering from a chronic disease. However, relapses could be seen months after the initial symptoms. The most common complications occasionally seen in the undulant and chronic forms are arthritis, spondylitis, meningitis and chronic fatigue (Hendaus et al., 2015; Bosilkovski et al., 2007; Morata et al., 2003).

*Author for correspondence; E-mail: ascientific@aec.org.sy
In mice, the most infected organs are spleen and liver which are rapidly colonised with Brucella in splenectomised mice. During the chronic phases, the higher Brucella colonisation of the spleen protects the liver from infection (Grilló et al., 2012). Therefore, spleen is the best target organ to study Brucella infections. Chronic steady phase, where bacterial counts were stable, commonly started at 21 days and lasting till 56-77 days post-infection. At this phase, the maximum number of CFU within spleen is recorded. On the contrary, a slow elimination of the bacteria from target organs is observed during the chronic declining phase which may last beyond 252 days (High et al., 2007). However, experiments on mice are not normally prolonged beyond 120 days. Large and prominent splenomegaly was rarely seen in ruminants (CarvalhoNeta et al., 2010) and the sequelae observed in chronic brucellosis in humans have not been reported in mice (Bosilkovski et al., 2007).

Although many regimens have been experimented and tested, treatment regimen approved by the World Health Organisation (1986) still recommended for the treatment of human brucellosis. However, the incidence of treatment failure and frequent relapses are still a major concern (Roushan et al., 2006). Moreover, quinolones utilisation as alternatives in the treatment of brucellosis did not improve the results (Lopez-Merino et al., 2004). 
Currently the human chronic brucellosis is generally treated with antibiotic triple therapy. The combination of rifampin, doxycycline, and streptomycin often is used whereas in non-responded or complicated cases, quinolones could be added to the conventional treatment as a third agent (Falagas and Bliziotis, 2006).

The aim of the current study was to compare the shortterm treatment efficacy of several quinolones alone or in combination with doxycycline in experimentally induced B. melitensis $16 \mathrm{M}$ infection in mice.

\section{Materials and Methods}

Bacteria. Brucella melitensis 16M strain was obtained from the Laboratory of Microbiology and Immunology URBM (University of Namur, Belgium) and grown in the Department of Molecular Biology and Biotechnology (Atomic Energy Commission of Syria), for $48 \mathrm{~h}$ in $2 \mathrm{YT}$ agar (peptone, $16 \mathrm{~g} / \mathrm{L}$; yeast extract, $10 \mathrm{~g} / \mathrm{L} ; \mathrm{NaCl}, 5 \mathrm{~g} / \mathrm{L}$; agar, $13 \mathrm{~g} / \mathrm{L}$ [GibcoBRL]) at $37^{\circ} \mathrm{C}$. Prior to dilution to appropriate concentrations of inoculates, bacteria were harvested into $20 \mathrm{~mL}$ of sterile phosphate-buffered saline (PBS) and the bacterial suspension was standardized to $10^{10}$ colony-forming units $(\mathrm{CFU}) / \mathrm{mL}$. The concentrations were determined retrospectively by enumeration of ten-fold dilutions of the inoculates on 2YT plates. All experiments with live Brucella were performed in biosafety level 2 facilities.

Antibiotics. Doxycycline (Sigma, St. Louis, USA), ciprofloxacin (Bayer, Istambul, Turkey), ofloxacin (Sigma), and levofloxacin (Sigma) were dissolved according to the manufacturer's recommendations to a working concentration of $8 \mathrm{mg} / \mathrm{mL}$. Antibiotics were prepared freshly every day and sterilized through a $0.2 \mu \mathrm{m}$ filter.

Animals. The effect of quinolones against chronic Brucella infection was studied according to the method of Domingo et al. (1995) with some modifications. Briefly, between February and October 2012, 200 BALB/c mice were infected with $\approx 10^{6} \mathrm{CFU}$ of $B$. melitensis $16 \mathrm{M}$ intraperitoneally. Thirty days later, the infected mice were divided into 8 groups of 25 mice each. The untreated control group received only the vehicle and was included in order to estimate the normal splenic infection during the course of this study, 225 days. At the $31^{\text {st }}$ day, the seven other groups received one of the following treatment regimens: doxycycline (oral, $40 \mathrm{mg} / \mathrm{kg}$ every $12 \mathrm{~h}$ for 14 days); ciprofloxacin, ofloxacin or levofloxacin (oral, $40 \mathrm{mg} / \mathrm{kg} / \mathrm{day}$ of body weight for 14 days); and one of doxycycline-ciproflox-acin, doxycycline-ofloxacin or doxycycline-levofloxacin combinations at the same doses mentioned above (i.e. doxycycline orally, $40 \mathrm{mg} / \mathrm{kg}$ every $12 \mathrm{~h}$ for 14 days; and quinolone orally, $40 \mathrm{mg} /$ $\mathrm{kg}$ /day of body weight for 14 days). The experimental procedures on mice and the facilities used to hold the experimental animals are in accordance to National (Real Decreto 233/1988, in BOE number 67) law. Batches of five mice were sacrificed by cervical dislocation, on 45, 90, 135, 180 and 225 days post-infection. At post-mortem, spleens were removed, weighted, homogenised in $3 \mathrm{~mL}$ of distilled water using a stomacher 80-Biomaster (Seward, England). Bacterial loads were determined following enumeration of ten-fold serial dilutions on 2YT agar plates (incubated for 3 days at $37{ }^{\circ} \mathrm{C}$ in air, with $10 \% \mathrm{CO}_{2}$ ) using the spread plate technique. The average of three replicates of each dilution was calculated, and considered as the mean $\mathrm{CFU} / \mathrm{mL}$ of each studied mouse.

Statistical analysis. Data were transformed into $\log _{10}$ CFU. Differences in CFU between the treated and untreated groups were evaluated by student's t-test. The data was analysed by version $5.0 \mathrm{GraphPad}$ Prism. P values of 0.05 or less were considered statistically significant.

\section{Results and Discussion}

The results show that doxycycline was effective at day 45 post-infection $(\mathrm{p}=0.03)$ when compared with the control. Doxycycline and ofloxacin were found the most effective at day 90 post-infection $(\mathrm{p}<0.0001$ and $\mathrm{p}=0.005$, respectively). Whereas, ciprofloxacin and levofloxacin were less effective ( $\mathrm{p}=0.011$ and $\mathrm{p}=0.047$, respectively). Only doxycycline was found effective at day 135 post-infection $(\mathrm{p}<0.001)$. In the same way, doxycycline was more effective than ciprofloxacin, ofloxacin and levofloxacin at day 90 post-challenge $(\mathrm{p}<0.0001, \mathrm{p}<0.0005$ and $\mathrm{p}<0.0001$, respectively); and most effective than ciprofloxacin and ofloxacin at day 135 post-infection ( $\mathrm{p}=0.04$ and $\mathrm{p}=0.02$, respectively) as shown in Fig. 1. Furthermore, Fig. 2 indicates that doxycycline-ciprofloxacin, doxycycline-ofloxacin and doxycycline-levofloxacin combinations were extremely efficient at day 45 post-challenge ( $<<0.0001$ at all cases); and doxycycline-ciprofloxacin and doxycyclineofloxacin combinations were very effective at day 90 post-challenge $(\mathrm{p}=0.0026$ and $\mathrm{p}=0.0015$, respectively). 
Additionally, doxycycline-ciprofloxacin combination was very efficient at day 135 post-infection $(\mathrm{p}<0.0001)$, and doxycycline-ofloxacin combination was less effective $(\mathrm{p}=0.032)$. However, comparing with doxycycline, splenic CFU reduction was observed from day 45 postinfection when using all doxycycline-quinolone combinations $(p<0.0001)$ comparing with doxycyclineciprofloxacin and doxycycline-ofloxacin combinations; and $\mathrm{p}=0.024$ comparing with doxycycline-levofloxacin combination.

The success of any drug in the treatment of brucellosis depends on its ability to penetrate the phagocytic cell membrane and work within its acidic environment. The failure in the treatment of acute cases of brucellosis may lead to the development of relapse, focal complications or even chronic disease (Castaño and Solera, 2009).

In human, most patients usually recover after one year of effective treatment while a few patients complain from focal or non-focal disease. Domingo et al. (1995) reviewed that relapses after the treatment with doxycycline alone for 21 days were higher than that noticed after the treatment with doxycycline for 45 days (29\% versus $10-14 \%$ ). In addition, ciprofloxacin at a $500 \mathrm{mg}$ dose twice a day within 20 days in chronic stage of disease essentially reduced duration of local inflammatory processes of brucellosis with simultaneous treatment of the chronic infection focus, provides good proximate and distant outcomes of treatment (Pappas et al., 2005).

Whereas, in mice, treatment regimens with azithromycin $50 \mathrm{mg} / \mathrm{kg} /$ day for 10 days, doxycycline $50 \mathrm{mg} /$ $\mathrm{kg} / 12 \mathrm{~h}$ for 21 days or doxycycline $50 \mathrm{mg} / \mathrm{kg} / 12 \mathrm{~h}$ for 45 days, produced a significant reduction in the chronic infection. In addition, when doxycycline was administered for 45 days, it was possible to eradicate B. melitensis completely in comparison with the treatment for 21 days only (Domingo et al., 1995). In agreement with these results, present research results revealed that the short-term treatment with doxycycline for 14 days was more effective than all quinolones and almost completely eradicates the infection 135 days after infection. Similarly, Shasha et al. (1992) reported that the longer the treatment with doxycycline the better the results obtained.

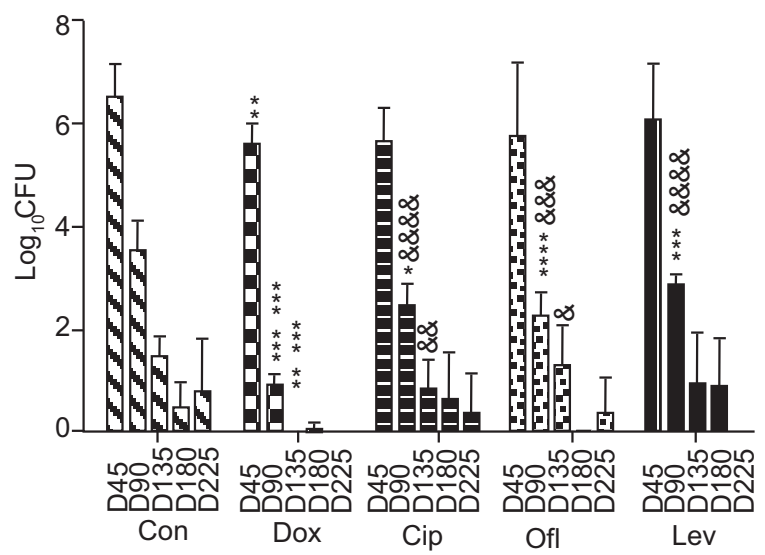

Fig. 1. Efficacy of doxycycline (Dox), ciprofloxacin (Cip), ofloxacin (Ofl) and levofloxacin (Lev) against $B$. melitensis $16 \mathrm{M}$ chronic infection. Each bar is representative of the mean bacterial count, counted in different days, in spleens of five mice \pm the standard error. ${ }^{*} \mathrm{p}=0.011, * * \mathrm{p}=0.03,{ }^{* * *} \mathrm{p}=0.047$, $* * * * \mathrm{p}=0.005, * * * * * \mathrm{p}=0.001$ and $* * * * * * \mathrm{p}<$ 0.0001 versus control (Con); whereas, ${ }_{p}=0.02,{ }^{\& \&} \mathrm{p}=0.04,{ }^{\& \& \&} \mathrm{p}<0.0005$ and ${ }^{\text {\&\&\& }} \mathrm{p}<$ 0.0001 versus doxycycline.

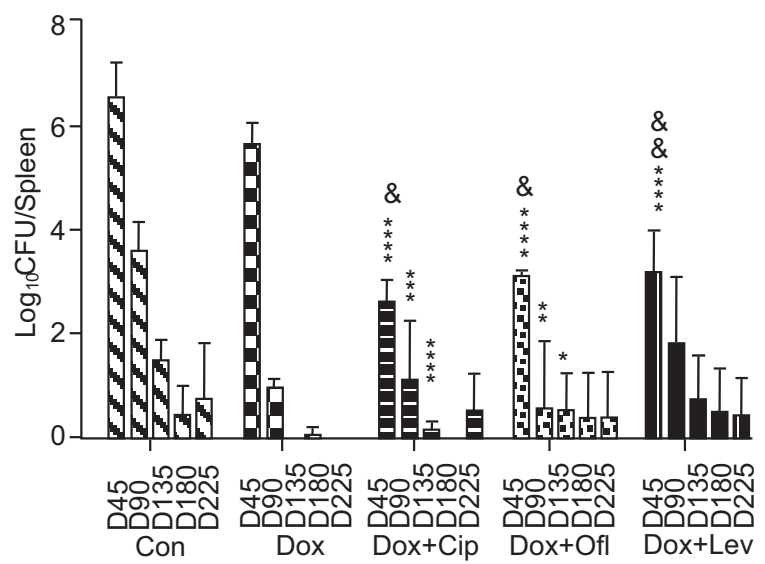

Fig. 2. Efficacy of doxycycline-ciprofloxacin (Dox+Cip), doxycycline-ofloxacin (Dox+Ofl) and doxycycline-levofloxacin (Dox+Lev) combinations against $B$. melitensis $16 \mathrm{M}$ chronic infection. Each bar is representative of the mean bacterial count, counted in different days, in spleens of five mice \pm the standard error. ${ }^{*} \mathrm{p}=0.032,{ }^{* *} \mathrm{p}=0.0015$, $* * * \mathrm{p}=0.0026$ and $* * * * \mathrm{p}<0.0001$ versus control (Con); whereas, ${ }^{\&} p<0.0001$ and $\& \& \mathrm{p}=0.024$ versus doxycycline (Dox). 
In addition, our results showed that ofloxacin, given orally for 14 days, was the most effective quinolone against chronic infection with $B$. melitensis $16 \mathrm{M}$ in murine model, and showed a significant reduction of the splenic infection at 90 days after infection. Moreover, doxycycline-quinolone combinations were more effective than the treatment with doxycycline alone since they could significantly reduce the splenic infection from day 45 post-infection. Unfortunately, these combinations could not completely eradicate the chronic infection.

Despite a few elevations in bacterial counts at 180 days post-infection comparing with that noticed at 135 days post-infection when doxycycline alone was applied; this elevation was not significant.

\section{Conclusion}

Different treatment methods were studied to control chronic human infection caused by Brucella. In conclusion, experiments on mice showed that short-term treatment with doxycycline was more effective than the treatment with quinolones. In addition, doxycyclinequinolones combinations were slightly more effective than doxycycline alone. However, treatment with doxycycline alone eliminates more number of bacteria than all other combinations after 135 days postinfection.

\section{Acknowledgement}

The authors would like to thank the Director General of the Atomic Energy Commission Syria (AECS) and the Head of the Molecular Biology and Biotechnology Department for their support.

\section{References}

Ariza, J., Pigrau, C., Cañas, C., Marrón, A., Martínez, F., Almirante, B., Corredoira, J.M., Casanova, A., Fabregat, J., Pahissa, A. 2001. Current understanding and management of chronic hepatosplenic suppurative brucellosis. Clinical Infectious Diseases, 32: 1024-1033.

Bosilkovski, M., Krteva, L., Dimzova, M., Kondova, I. 2007. Brucellosis in 418 patients from the Balkan Peninsula: exposure-related differences in clinical manifestations, laboratory test results, and therapy outcome. International Journal of Infectious Diseases, 11: $342-347$.
Castaño, M.J., Solera, J. 2009. Chronic brucellosis and persistence of Brucella melitensis DNA. Journal of Clinical Microbiology, 47: 2084-2089.

Carvalho-Neta, A.V., Mol, J.P.S., Xavier, M.N., Paixão, T.A., Lage, A.P., Santos, R.L. 2010. Pathogenesis of bovine brucellosis. Veterinary Journal, 184: 146-155.

Díaz, R., Ariza, J., Alberola, I., Casanova, A., Rubio, M.F. 2006. Secondary serological response of patients with chronic hepatosplenic suppurative brucellosis. Clinical and Vaccine Immunology, 13: 1190-1196.

Domingo, S., Gastearena, I., Vitas, A.I., López-Goñi, I., Dios-Viéitez, C., Díaz, R., Gamazo, C. 1995. Comparative activity of azithromycin and doxycycline against Brucella spp. infection in mice. Journal of Antimicrobial Chemotherapy, 36: 647-656.

Falagas, M.E., Bliziotis, I.A. 2006. Quinolones for treatment of human brucellosis: critical review of the evidence from microbiological and clinical studies. Antimicrobial Agents and Chemotherapy, 50: 22-33.

Grilló, M.J., Blasco, J.M., Gorvel, J.P., Moriyón, I., Moreno, E. 2012. What have we learned from brucellosis in the mouse model. Veterinary Research, 43: 29-63.

Hendaus, M.A., Qaqish, R., Alhammadi, A.H. 2015. Neurobrucellosis in children. Asian Pacific Journal of Tropical Biomedicine, 5: 158-161.

High, K.P., Prasad, R., Marion, C.R., Schurig, G.G., Boyle, S.M., Sriranganathan, N. 2007. Outcome and immune responses after Brucella abortus infection in young adult and aged mice. Biogerontology, 8: 583-593.

Lopez-Merino, A., Contreras-Rodriguez, A., MigranasOrtiz, R., Orrantia-Gradin, R., Hernandez-Oliva, G.M., Gutierrez-Rubio, A., Cardeñosa, T. 2004. Susceptibility of Mexican Brucella isolates to moxifloxacin, ciprofloxacin and other antimicrobials used in the treatment of human brucellosis. Scandinavian Journal of Infectious Diseases, 36: 636-638.

Morata, P., Queipo-Ortuño, M.I., Reguera, J.M., García-Ordoñez, M.A., Cárdenas, A., Colmenero, J.D. 2003. Development and evaluation of a PCR-enzyme-linked immunosorbent assay for diagnosis of human brucellosis. Journal of Clinical 
Microbiology, 41: 144-148.

Pappas, G., Papadimitriou, P., Akritidis, N., Christou, L., Tsianos, E.V. 2006. The new global map of human brucellosis. Lancet Infectious Diseases, 6: 91-99.

Pappas, G., Solera, J., Akritidis, N., Tsianos, E. 2005.

New approaches to the antibiotic treatment of brucellosis. International Journal of Antimicrobial Agents, 26: 101-105.

Roushan, M.R.H., Mohraz, M., Hajiahmadi, M., Ramzani, A., Valayati, A.A. 2006. Efficacy of gentamycin plus doxycycline versus streptomycin plus doxycycline in the treatment of brucellosis in humans. Clinical Infectious Diseases, 42: 10751080.

Shasha, B., Lang, R., Rubinstein, E. 1992. Therapy of experimental murine brucellosis with streptomycin, co-trimoxazole, ciprofloxacin, floxacin, pefloxacin, doxycycline and rifampicin. Antimicrobial Agents and Chemotherapy, 36: 973-976.

WHO, 1986. Joint FAO/WHO Expert Committee on Brucellosis, World Health Organization Report Series, 740: 1-132. 